\title{
Effective three-band structure in Fe-based superconductors
}

\author{
David Möckli* and E.V.L. de Mello ${ }^{\dagger}$ \\ Instituto de Física da Universidade Federal Fluminense - CEP 24.210-346 - Niterói - RJ, Brazil
}

(Dated: September 13, 2021)

\begin{abstract}
We present self-consistent calculations of the multi-gap structure measured in some Fe-based superconductors. These materials are known to have structural disorder in real space and a multigap structure due to the $3 d$ Fe-orbitals contributing to a complex Fermi surface topology with hole and electron pockets. Different experiments identify three $s$-wave like superconducting gaps with a single critical temperature $\left(T_{c}\right)$. We investigate the temperature dependence of these gaps by a multiband Bogoliubov-deGennes theory at different pockets in the presence of effective hybridizations between some bands and an attractive temperature dependent intra-band interaction. We show that this approach reproduces the three observed gaps and single $T_{c}$ in different compounds of $\mathrm{Ba}_{1-x} \mathrm{~K}_{x} \mathrm{Fe}_{2} \mathrm{As}_{2}$, providing some insights on the inter-band interactions.
\end{abstract}

PACS numbers: 74.20.De,74.20.Mn, 74.70.Xa

Keywords: electronic inhomogeneity, Fe-based superconductors, multi-gap superconductivity, BdG theory.

\section{INTRODUCTION}

First principle band theory calculations, such as local density approximation (LDA) [1-3] on $\mathrm{Ba}_{1-x} \mathrm{~K}_{x} \mathrm{Fe}_{2} \mathrm{As}_{2}$, predicted five bands of the Fe 3 d orbitals across the Fermi surface (FS) forming three hole-like FSs centered at the zone center and two electron-like FSs centered at the zone corner. This Fe-based superconductor (FeSC) revealed to be a highly complex high critical temperature $\left(T_{c}\right)$ superconducting (SC) material with multi-band and multi-gap structure [4]. Furthermore, it also possess an unusual anisotropy in the ab-plane resistivity [5-7] just above $T_{c}$, possibly related to an electronic phase separation transition [8] or a nematic phase [9].

Experiments such as angle-resolved photoemission spectroscopy (ARPES) [4, 10, 11], point-contact Andreev reflection spectroscopy (PCAR) [12-14] and muon-spin rotation ( $\mu \mathrm{SR})[15]$ identify two or more nodeless $s$-wave like SC gaps. Here we want to address the classical ARPES experiment that identified nearly two coinciding gap structures in the $\alpha$ and $\gamma$, and another one in the large $\beta$ pocket of $\mathrm{Ba}_{1-x} \mathrm{~K}_{x} \mathrm{Fe}_{2} \mathrm{As}_{2}$ with $T_{c}=38 \mathrm{~K}$ $[4,16]$. Differently than PCAR, ARPES is able to connect the gaps with the pockets or bands in the Brillouin zone.

Earlier it was predicted by Suhl et al [17] that for the case in which two different bands develop SC gaps with different values, and in the absence of interband interaction, two $T_{c}$ 's exist. A small amount of interband scattering makes the two to merge to a single $T_{c}$. This result suggests a clear interaction between the bands forming the hole and electron pockets in the $\mathrm{Ba}_{1-x} \mathrm{~K}_{x} \mathrm{Fe}_{2} \mathrm{As}_{2}$ system. An additional difficulty to treat this system is the multi-orbital character of the bands or pockets at the FS [18] instead of the single structure like the $s$ - $d$ superconductors [17].

\footnotetext{
*dmockli@if.uff.br

† evandro@if.uff.br
}

The main point tackled here concerns the $k$-dependent hybridization among the multi-bands forming the pockets where SC gaps were measured by ARPES [4, 16]. Since the bands forming the pockets can be model by two [19] or more orbitals [18], we describe the inter-band scattering by the hybridization of these orbitals. This generates a $k$-dependent interaction arising from a nonlocal character of the mixing [20]. In the real calculations we consider a special case of a local constant multi-orbital hybridization between the $\alpha-\beta$ and $\beta-\gamma$ pockets, representing an average over the Brillouin zone.
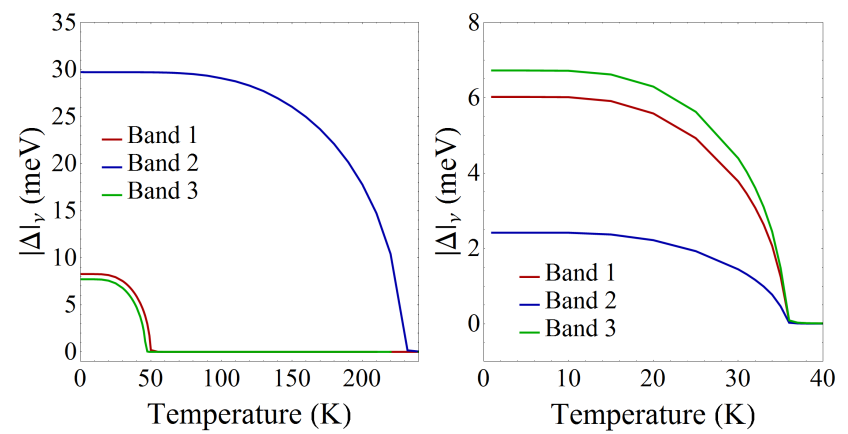

FIG. 1: The left panel shows the SC gaps on three non-interacting bands with the same band parameters of the right panel extracted from [4] and [16]. The big difference is the inter-band hopping. Bands 1 (red), 2 (blue) and 3 (green) have the same hoppings as the $\alpha, \beta$ and $\gamma$ pockets respectively listed in table I.

\section{MULTI-BAND SUPERCONDUCTING THEORY}

As mentioned, LDA calculations [1-3] with the $\mathrm{Ba}_{1-x} \mathrm{~K}_{x} \mathrm{Fe}_{2} \mathrm{As}_{2}$ family yielded five multi orbital bands across the FS. On the other hand, ARPES experiments $[4,16]$ measured three $s$-wave like $\mathrm{SC}$ gaps at different 
pockets with quite different low temperature intensity and the same $T_{c}$. This behavior is likely to occur due to interband interactions [17]. Thus, we propose the following mean field Hamiltonian, with two terms, namely

$$
\mathcal{H}_{\mathrm{MF}}=\mathcal{H}_{\text {intra }}+\mathcal{H}_{\text {inter }}
$$

where $\mathcal{H}_{\text {intra }}$ contains the information of the three separate hole and electron pockets $\alpha, \beta$ and $\gamma[4,16]$,

$$
\mathcal{H}_{\text {intra }}=\sum_{\mathbf{i} \neq \mathbf{j}} \sum_{\nu=\alpha, \beta, \gamma} \sum_{\sigma} t_{\nu}(\mathbf{i}, \mathbf{j}) c_{\nu \sigma}^{\dagger}(\mathbf{i}) c_{\nu \sigma}(\mathbf{j})-\sum_{\mathbf{i}, \nu, \sigma} \tilde{\mu}_{\nu}(\mathbf{i}) c_{\nu \sigma}^{\dagger}(\mathbf{i}) c_{\nu \sigma}(\mathbf{i})+\sum_{\mathbf{i} \mathbf{j}, \nu}\left[\Delta_{\nu}(\mathbf{i}, \mathbf{j}) c_{\nu \uparrow}^{\dagger}(\mathbf{j}) c_{\nu \downarrow}^{\dagger}(\mathbf{i})+\text { h.c. }\right] .
$$

The $t_{\nu}(\mathbf{i}, \mathbf{j})$ are intra-band hoppings between lattice sites $\mathbf{i}$ and $\mathbf{j}$ up to second nearest neighbors as derived by the ARPES band dispersion [16], based on their results and on the theory of magnetic excitation on a four orbital model [21]. We introduced a short-hand notation for the shifted chemical potential $\tilde{\mu}_{\nu}(\mathbf{i})=\mu_{\nu}(\mathbf{i})-U_{\nu}(\mathbf{i}) \rho_{\nu}(\mathbf{i}) / 2$, which includes: the local chemical potential $\mu_{\nu}(\mathbf{i})$, the onsite Coulomb repulsion $U_{\nu}(\mathbf{i})$, that due to the mean-field treatment just enters as a rescaling factor, and the band charge density $\rho_{\nu}(\mathbf{i})$. The spin index $\sigma$ assumes either $\uparrow$ or $\downarrow$, and the creation and annihilation operators obey the Fermi anti-commutation relation $\left\{c_{\mu \sigma}(\mathbf{i}), c_{\nu \sigma^{\prime}}^{\dagger}(\mathbf{j})\right\}=$ $\delta_{\mathbf{i j}} \delta_{\mu \nu} \delta_{\sigma \sigma^{\prime}}$

The second term in equation (1) includes the interband contribution along the same lines of the theory developed by Kishore et al. [22, 23], and Caixeiro et al. [20], where the hybridization between overlapping bands near the Fermi surface is approximated by a constant (representing an average over the Brillouin zone) nearest neighbour hopping

$$
\mathcal{H}_{\text {inter }}=\sum_{\mathbf{i} \neq \mathbf{j}} \sum_{\mu \neq \nu} \sum_{\sigma} t_{\mu \nu}(\mathbf{i}, \mathbf{j}) c_{\mu \sigma}^{\dagger}(\mathbf{i}) c_{\nu \sigma}(\mathbf{j})
$$

The band density $\rho_{\nu}(\mathbf{i})$ is self-consistently regulated by adjusting $\mu_{\nu}(\mathbf{i})$ until $\rho_{\nu}(\mathbf{i})$ converges to some fixed value. Due to its local expression this approach can be applied

to cases where there is some degree of disorder in the electronic density.

The local SC band gap is $\Delta_{\nu}(\mathbf{i}, \mathbf{j})=$ $-V(T)\left\langle c_{\nu \downarrow}(\mathbf{i}) c_{\nu \uparrow}(\mathbf{j})\right\rangle$, where $\langle\cdots\rangle$ represents a thermal average. $V(T)$ is a temperature dependent potential derived from a two-phase separated system. Using a typical Ginzburg-Landau free energy expansion it is possible to show that free energy barrier between the two phases is proportional to $\left(T_{\mathrm{PS}}-T\right)^{2}$, where $T_{\mathrm{PS}}$ is the phase separation critical temperature. This approach has been applied to cuprates and in this case $T_{\mathrm{PS}}$ is associated with the pseudogap temperature $T^{*}[24,25]$. As mentioned, there is experimental evidence that the $\mathrm{Ba}_{1-x} \mathrm{~K}_{x} \mathrm{Fe}_{2} \mathrm{As}_{2}$ system may also undergo an electronic phase separation [8] or nematic order [9]. Based on these observations, we take

$$
V(T)=-\left|V_{0}\right|\left(1-\frac{T}{T_{\mathrm{PS}}}\right)^{2}
$$

The Hamiltonian defined by (1) is diagonalized by the unitary Bogoliubov transformation

$$
c_{\nu \sigma}(\mathbf{i})=\sum_{n}\left(u_{n \nu}(\mathbf{i}) \gamma_{n \sigma}-\operatorname{sgn}(\sigma) v_{n \nu}^{*}(\mathbf{i}) \gamma_{n,-\sigma}^{\dagger}\right)
$$

which leads to the multi-band Bogoliubov-deGennes (BdG) equation

$$
E_{n}\left[\begin{array}{l}
u_{n \mu}(\mathbf{i}) \\
v_{n \mu}(\mathbf{i})
\end{array}\right]=\sum_{\mathbf{j}}\left[\begin{array}{cc}
\sum_{\nu}\left(t_{\mu \nu}(\mathbf{i}, \mathbf{j})-\tilde{\mu}_{\nu}(\mathbf{i}) \delta_{\mu \nu}\right) & \Delta_{\mu}(\mathbf{i}, \mathbf{j}) \delta_{\mu \nu} \\
\Delta_{\mu}^{*}(\mathbf{i}, \mathbf{j}) \delta_{\mu \nu} & -\sum_{\nu}\left(t_{\mu \nu}^{*}(\mathbf{i}, \mathbf{j})+\tilde{\mu}_{\nu}(\mathbf{i}) \delta_{\mu \nu}\right)
\end{array}\right]\left[\begin{array}{l}
u_{n \nu}(\mathbf{j}) \\
v_{n \nu}(\mathbf{j})
\end{array}\right]
$$

where $t_{\nu \nu} \equiv t_{\nu}$ in equation (6). The full BdG matrix has dimension $6 N^{2} \times 6 N^{2}$ in the three-band case, where $N \times N$ is the lattice's dimension. The positive quasiparticle excitations $\left\{E_{n}\right\}$ are used to self-consistently calculate the temperature dependent local $s$-wave gap for each band

$$
\Delta_{\nu}(\mathbf{i})=-V_{\nu}(\mathbf{i}) \sum_{n} u_{n \nu}(\mathbf{i}) v_{n \nu}^{*}(\mathbf{i}) \tanh \left(\frac{E_{n}}{2 k_{B} T}\right)
$$

and the local band density $\rho_{\nu}(\mathbf{i})=\sum_{\sigma}\left\langle c_{\nu \sigma}^{\dagger}(\mathbf{i}) c_{\nu \sigma}(\mathbf{i})\right\rangle$ as

$$
\rho_{\nu}(\mathbf{i})=2 \sum_{n}\left(\left|u_{n \nu}(\mathbf{i})\right|^{2} f_{n}+\left|v_{n \nu}(\mathbf{i})\right|^{2}\left(1-f_{n}\right)\right)
$$

where $f_{n}$ is the Fermi distribution of quasi-particles. Equation (7) allows for the simultaneous determination of $\Delta_{\alpha}(T), \Delta_{\beta}(T)$, and $\Delta_{\gamma}(T)$.

A typical solution of equation (7) with a single constant attractive potential for all three bands with independent 
band dynamics (no inter-band hoppings) is shown on the left panel of figure 1. The intra-band hoppings are those derived from Ding et al [16] that reproduces the three $\alpha$, $\beta$ and $\gamma$ pockets and are listed in table $\mathrm{I}$.

Notice that lower intra-band hoppings or lower kinetic energies yield bigger gaps and larger $T_{c}$ 's. However, as predicted by Suhl et al. [17] for two bands, non-zero inter-band hoppings modify the three-gap structure drastically as shown on the right panel of figure 1. The crucial difference in the second case is a single value for $T_{c}$ for all three bands, in accordance with experimental observations $[4,10-14]$.

\section{TEMPERATURE DEPENDENCE OF THE THREE-GAP STRUCTURE}
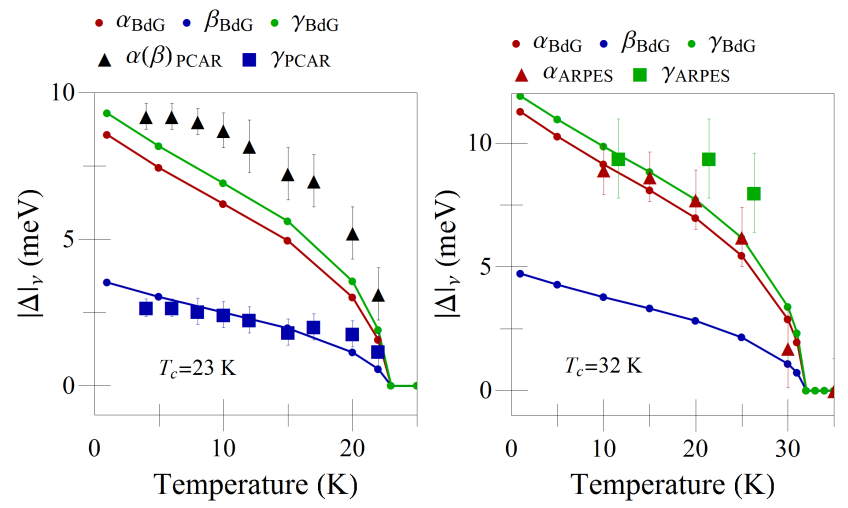

FIG. 2: On the left panel we show the theoretical BdG curves compared to PCAR $[13,14]$ for $\mathrm{Ba}_{1-x} \mathrm{~K}_{x} \mathrm{Fe}_{2} \mathrm{As}_{2}$ with $T_{c}=23 \mathrm{~K}$. PCAR identifies two SC bands that may correspond to the nearly coinciding $\alpha$ and $\gamma$ pocket identified by ARPES. On the right panel we show the BdG curves compared to the three bands from ARPES [10] for the compound with $T_{c}=32 \mathrm{~K}$. Reference [10] only estimates gap values for the $\beta$ pocket.

Now we apply multi-band BdG theory to model three compounds of $\mathrm{Ba}_{1-x} \mathrm{~K}_{x} \mathrm{Fe}_{2} \mathrm{As}_{2}$ with $T_{c}=23,32$ and 38 $\mathrm{K}$ to reproduce the $\mathrm{SC}$ gaps measured by different techniques. PCAR techniques [13, 14] find two SC bands, whereas ARPES [4] detects SC gaps in three bands or pockets in the Brillouin zone. It is possible that the bigger gap observed by PCAR may correspond to the $\alpha$ and $\gamma$ bands from ARPES, since they have about the same intensity.

For each compound we solve a three-band $6 N^{2} \times 6 N^{2}$ BdG matrix in a square lattice with periodic boundary conditions. Following the LDA calculations [1-3] and the derived bands by ARPES[16] we can obtain an estimation of the inter-band hoppings shown in the first three columns of table I. For instance, there is very little overlap between and no intersection between $\alpha$ and $\gamma$ and so we take $t_{\alpha \gamma}=0$ and larger inter-band hoppings to reflect band intersections between the $\alpha$ and $\beta$ and $\beta$
TABLE I: First and second nearest neighbour intra $t_{\nu}$ and inter-band hoppings $t_{\mu \nu}$ in meV used in the present calculations. Intra-band hoppings were extracted from ARPES and inter-band hoppings closely agree with band dispersion [16]. The parameters of this table are used for all three compound discussed in this paper.

\begin{tabular}{lllllll}
\hline \hline Hoppings $(\mathrm{meV})$ & $t_{\alpha}$ & $t_{\beta}$ & $t_{\gamma}$ & $t_{\alpha \beta}$ & $t_{\alpha \gamma}$ & $t_{\beta \gamma}$ \\
\hline $1^{\text {st }}$ & 160 & 13 & 380 & 165 & 0 & 140 \\
$2^{\text {ond }}$ & -52 & 42 & 80 & 0 & 0 & 0 \\
\hline
\end{tabular}

TABLE II: Phenomenological values of the electronic phase transition temperature $T_{\mathrm{PS}}$ and potential $\left|V_{0}\right|$ in equation (4) used to model $\mathrm{Ba}_{1-x} \mathrm{~K}_{x} \mathrm{Fe}_{2} \mathrm{As}_{2}$ with different $T_{c}$ 's.

\begin{tabular}{llll}
\hline \hline Compound & $T_{c}(\mathrm{~K})$ & $T_{\mathrm{PS}}(\mathrm{K})$ & $\left|V_{0}\right|(\mathrm{meV})$ \\
\hline $\mathrm{Ba}_{0.6} \mathrm{~K}_{0.4} \mathrm{Fe}_{2} \mathrm{As}_{2}$ & 38 & 165 & -400 \\
$\mathrm{Ba}_{1-x} \mathrm{~K}_{x} \mathrm{Fe}_{2} \mathrm{As}_{2}$ & 32 & 130 & -360 \\
$\mathrm{Ba}_{1-x} \mathrm{~K}_{x} \mathrm{Fe}_{2} \mathrm{As}_{2}$ & 23 & 80 & -300 \\
\hline \hline
\end{tabular}

and $\gamma$ pockets $[16,27]$, which are shown in the last three columns of table I.

To model the experiment results we perform calculations with these hoppings listed in table I, used for all three compounds. The charge distribution in each pocket $\left(\rho_{\alpha}, \rho_{\beta}, \rho_{\gamma}\right)=(0.192,0.084,0.12)$ are proportional to specific Fermi areas [27], and Fermi velocities [4, 15]. Even if the system has a disordered electronic density, the average gap is the same of that of a homogeneous one with the same average density. Furthermore, ARPES and PCAR measure only the average gap.

To obtain the $\mathrm{SC}$ gaps of different $\mathrm{Ba}_{1-x} \mathrm{~K}_{x} \mathrm{Fe}_{2} \mathrm{As}_{2}$ compounds with distinct $T_{c}$ 's we used the band parameters discussed before and estimate the two-body attractive potential in equation (4) as listed in table II. The $\Delta_{\nu}(T)$ results for the compound with $T_{c}=23 \mathrm{~K}$ is depicted on the left panel of figure 2. The coloured solid lines are the theoretical BdG curves obtained from (7). We observe that PCAR $[13,14]$ (black triangles) scales more closely with the $\mathrm{BdG} \alpha / \gamma$ bands. In the case of the compound with $T_{c}=32 \mathrm{~K}$ we show also experimental points from ARPES [10] in comparison to the theoretical $\mathrm{BdG}$ curves on the right panel of figure 2. Experimental points for the $\beta$ bands were unavailable and estimated under $4 \mathrm{meV}$ [10], consistent with the blue $\beta$ BdG curve.

The results for $\mathrm{Ba}_{0.6} \mathrm{~K}_{0.4} \mathrm{Fe}_{2} \mathrm{As}_{2}$ with $T_{c}=38 \mathrm{~K}$ are shown in figure 3, with all bands (left panel) and with each band shown separately for clarity. Two different sets of ARPES points are displayed denoted by ARPES1 referring to [4] and ARPES2 according to [26]. It is interesting to note that the measured band's correspondent SC gaps are reproduced by a temperature-dependent attractive potential within a three-band BdG theory. This is indicated by the grey curves in figure 3, which correspond to the case where a constant potential that gives 

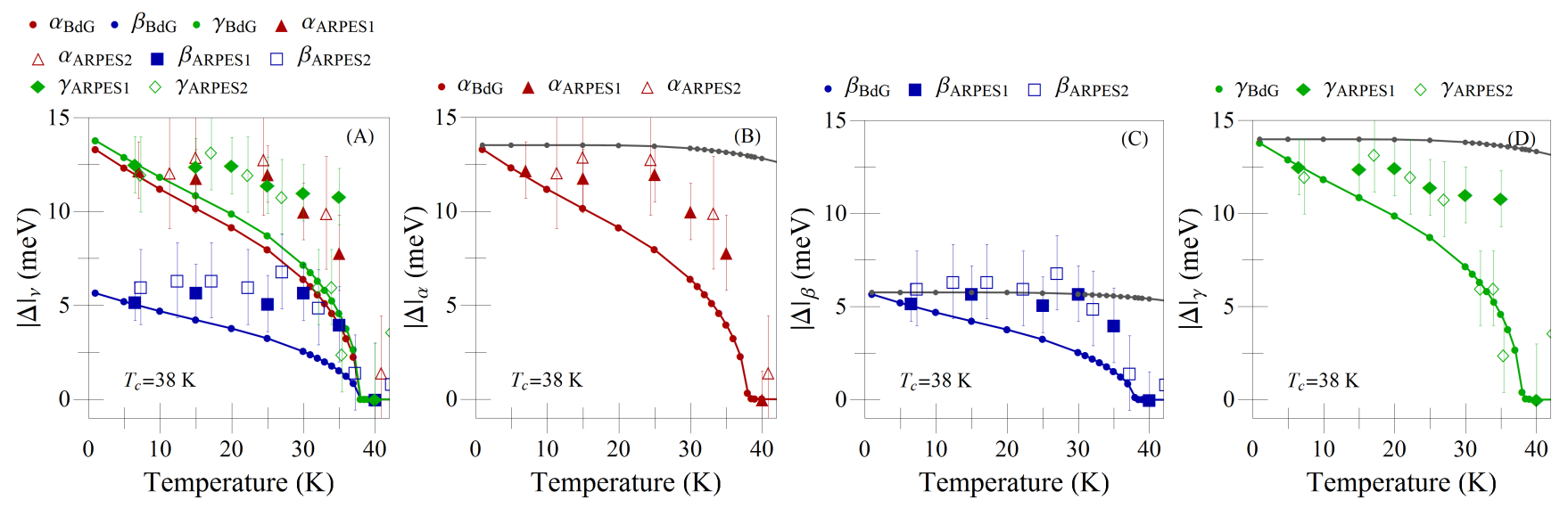

FIG. 3: Temperature dependence of (a) the jointly obtained SC gaps $\Delta_{\nu}(T)$ from multi-band BdG theory (solid lines) in comparison to references [4] (empty points) and [26] (solid points) for $\mathrm{Ba}_{0.6} \mathrm{~K}_{0.4} \mathrm{Fe}_{2} \mathrm{As}_{2}$ with $T_{c}=38 \mathrm{~K}$ and $N=8$. We show the three sets of data corresponding to (b) $\nu=\alpha$, (c) $\nu=\beta$, and (d) $\nu=\gamma$ separately for clarity. The grey curves correspond to the case of a constant potential, which is included for comparison.

the same $\Delta_{\nu}(T=0)$ was used.

Despite the real and $k$-space complexity of the system, we obtain a reasonable agreement with the experimental values, using a single attractive potential (equation (4)) for the three bands for each compound. The misfit of the experimental points with respect to the theoretical $\mathrm{BdG}$ curves may be due to differences in the tight binding hoppings and, more importantly, in the finite size used in the calculations with respect to the real system. Our matrix is only $384 \times 384$ and it is solved by exact diagonalization. New calculations using a more powerful method $[28,29]$ that allows investigation of much larger arrays are being presently considered. However, we believe that the essential properties of these compounds are captured by the present calculations.

\section{CONCLUSION}

We have discussed along the paper the evidence of electronic disorder complexity in real and $k$-space of the $\mathrm{Ba}_{1-x} \mathrm{~K}_{x} \mathrm{Fe}_{2} \mathrm{As}_{2}$ compounds. A possible electronic phase separation or a nematic order together with the multiorbital band structure make this system quite unusual. As expected the description to its SC multi-gap properties is not a simple task, unlike $\mathrm{MgB}_{2}$ with two bands and two conventional BCS-like gaps [30].
The SC multi-gap structure revealed by ARPES $[4,16]$ with three different gaps on distinct locations of the Brillouin zone, but with a single $T_{c}$, in light of the two band theory of Suhl et al [17], is an indication of inter-band interaction or hybridization. This was confirmed by selfconsistent calculations with the BdG theory with interband hoppings. These hoppings describe phenomenologically the interaction between bands, mediated by the hybridized iron orbitals.

The anisotropy in real space was also taken into consideration. In analogy with the cuprate superconductors which displayed many indications of charge disorder,[31, 32 ] we also use the observed charge disorder or nematic order as the origin of a two-body attractive pair potential with a temperature dependence derived from a GL theory.

We conclude that the multi-band scattering via multiorbital hybridizations and in plane charge disorder are well known important properties of high complexity of these materials that are essential to quantify the pairing amplitudes and the overall SC properties.

\section{ACKNOWLEDGMENTS}

We gratefully acknowledge partial financial aid from Brazilian agencies CAPES, FAPERJ and CNPq.
[1] D. J. Singh and M.-H. Du, Phys. Rev. Lett. 100, 237003 (2008).

[2] F. Ma and Z.-Y. Lu, Phys. Rev. B 78, 033111 (2008).

[3] G. Xu, H. Zhang, X. Dai, and Z. Fang, EPL (Europhysics Lett. 84, 67015 (2008).

[4] H. Ding, P. Richard, K. Nakayama, K. Sugawara, T. Arakane, Y. Sekiba, A. Takayama, S. Souma, T. Sato,
T. Takahashi, Z. Wang, X. Dai, Z. Fang, G. F. Chen, J. L. Luo, and N. L. Wang, EPL 83, 47001 (2008).

[5] J.-H. Chu, J. G. Analytis, K. De Greve, P. L. McMahon, Z. Islam, Y. Yamamoto, and I. R. Fisher, Science 329, 824 (2010).

[6] M. a. Tanatar, E. C. Blomberg, a. Kreyssig, M. G. Kim, N. Ni, a. Thaler, S. L. Budko, P. C. Canfield, a. I. Gold- 
man, I. I. Mazin, and R. Prozorov, Phys. Rev. B 81, 184508 (2010).

[7] J. J. Ying, X. F. Wang, T. Wu, Z. J. Xiang, R. H. Liu, Y. J. Yan, a. F. Wang, M. Zhang, G. J. Ye, P. Cheng, J. P. Hu, and X. H. Chen, Phys. Rev. Lett. 107, 067001 (2011).

[8] J. T. Park, D. S. Inosov, C. Niedermayer, G. L. Sun, D. Haug, N. B. Christensen, R. Dinnebier, A. V. Boris, A. J. Drew, L. Schulz, T. Shapoval, U. Wolff, V. Neu, X. Yang, C. T. Lin, B. Keimer, and V. Hinkov, Phys. Rev. Lett. 102, 117006 (2009).

[9] E. C. Blomberg, M. a. Tanatar, R. M. Fernandes, I. I. Mazin, B. Shen, H.-H. Wen, M. D. Johannes, J. Schmalian, and R. Prozorov, Nat. Commun. 4, 1914 (2013).

[10] D. V. Evtushinsky, D. S. Inosov, V. B. Zabolotnyy, A. Koitzsch, M. Knupfer, B. Büchner, M. S. Viazovska, G. L. Sun, V. Hinkov, A. V. Boris, C. T. Lin, B. Keimer, A. Varykhalov, A. A. Kordyuk, and S. V. Borisenko, Phys. Rev. B 79, 054517 (2009).

[11] P. Richard, T. Sato, K. Nakayama, T. Takahashi, and H. Ding, Reports Prog. Phys. 74, 124512 (2011).

[12] D. Daghero, M. Tortello, R. Gonnelli, V. Stepanov, N. Zhigadlo, and J. Karpinski, Phys. Rev. B 80, 060502 (2009).

[13] P. Szabó, Z. Pribulová, G. Pristáš, S. Budko, P. Canfield, and P. Samuely, Phys. Rev. B 79, 012503 (2009).

[14] P. Samuely, Z. Pribulová, P. Szabó, G. Pristáš, S. Budko, and P. Canfield, Phys. C Supercond. 469, 507 (2009).

[15] D. V. Evtushinsky, D. S. Inosov, V. B. Zabolotnyy, M. S. Viazovska, R. Khasanov, A. Amato, H.-H. Klauss, H. Luetkens, C. Niedermayer, G. L. Sun, V. Hinkov, C. T. Lin, A. Varykhalov, A. Koitzsch, M. Knupfer, B. Büchner, A. A. Kordyuk, and S. V. Borisenko, New J. Phys. 11, 055069 (2009).

[16] H. Ding, K. Nakayama, P. Richard, S. Souma, T. Sato, T. Takahashi, M. Neupane, Y.-M. Xu, Z.-H. Pan, A. V. Fedorov, Z. Wang, X. Dai, Z. Fang, G. F. Chen, J. L. Luo, and N. L. Wang, J. Phys. Condens. Matter 23, 135701 (2011).
[17] H. Suhl, B. Matthias, and L. Walker, Phys. Rev. Lett. 3, 552 (1959).

[18] P. J. Hirschfeld, M. M. Korshunov, and I. I. Mazin, Reports Prog. Phys. 74, 124508 (2011).

[19] M. Daghofer, A. Nicholson, A. Moreo, and E. Dagotto, Phys. Rev. B 81, 014511 (2010).

[20] E. S. Caixeiro and A. Troper, Phys. Rev. B 82, 014502 (2010).

[21] M. Korshunov and I. Eremin, Phys. Rev. B 78, 140509 (2008).

[22] R. Kishore and S. Joshi, Phys. Rev. B 2, 1411 (1970).

[23] G. Japiassú, M. Continentino, and A. Troper, Phys. Rev. B 45, 2986 (1992).

[24] E. V. L. de Mello, R. B. Kasal, and C. A. C. Passos, J. Phys. Condens. Matter 21, 235701 (2009).

[25] E. V. L. de Mello, EPL 98, 57008 (2012).

[26] L. Wray, D. Qian, D. Hsieh, Y. Xia, L. Li, J. G. Checkelsky, A. Pasupathy, K. K. Gomes, C. V. Parker, A. V. Fedorov, G. F. Chen, J. L. Luo, A. Yazdani, N. P. Ong, N. L. Wang, and M. Z. Hasan, Phys. Rev. B 78, 184508 (2008).

[27] T. Sato, K. Nakayama, Y. Sekiba, P. Richard, Y.-M. Xu, S. Souma, T. Takahashi, G. F. Chen, J. L. Luo, N. L. Wang, and H. Ding, Phys. Rev. Lett. 103, 047002 (2009).

[28] L. Covaci, F. M. Peeters, and M. Berciu, Phys. Rev. Lett. 105, 167006 (2010).

[29] A. Weisse, G. Wellein, A. Alvermann, and H. Fehske, Rev. Mod. Phys. 78, 275 (2006).

[30] Y. Wang, T. Plackowski, and A. Junod, Phys. C Supercond. 355, 179 (2001).

[31] R. Comin, A. Frano, M. M. Yee, Y. Yoshida, H. Eisaki, E. Schierle, E. Weschke, R. Sutarto, F. He, A. Soumyanarayanan, Y. He, M. Le Tacon, I. S. Elfimov, J. E. Hoffman, G. A. Sawatzky, B. Keimer, and A. Damascelli, Science 343, 390 (2014).

[32] E. H. da Silva Neto, P. Aynajian, A. Frano, R. Comin, E. Schierle, E. Weschke, A. Gyenis, J. Wen, J. Schneeloch, Z. Xu, S. Ono, G. Gu, M. Le Tacon, and A. Yazdani, Science 343, 393 (2014). 